\title{
Enhanced Recovery from Contraction-Induced Damage in Skeletal Muscles of Old Mice Following Treatment with the Heat Shock Protein Inducer 17-(Allylamino)-17-Demethoxygeldanamycin
}

\author{
Anna C. Kayani, Graeme L. Close, Caroline S. Broome, Malcolm J. Jackson, and Anne McArdle
}

\begin{abstract}
Unlike muscles of young mice, skeletal muscles of old mice fail to recover completely following contractioninduced damage. The mechanisms by which this occurs are not fully understood. The ability of muscles of old mice to adapt following exercise by the increased production of heat shock proteins (HSPs) is blunted. Studies using transgenic mice have shown that this inability to produce HSPs has a major effect on muscle regeneration. Overexpression of HSP70 facilitated complete recovery of maximum tetanic force generation in muscles of old transgenic mice following contraction induced-damage in comparison with a deficit in muscles of old wild-type (WT) mice. We hypothesized that pharmacological induction of HSP70 in muscles of old WT mice would result in enhanced recovery from contraction-induced damage. A single dose of $40 \mathrm{mg} / \mathrm{kg}$ of 17 -(allylamino)-17-demethoxygeldanamycin (17AAG) resulted in a significant increase in the HSP70 content of extensor digitorum longus muscles of adult C57BL6/J mice 3 days following treatment compared with vehicle-treated mice. Four weekly treatments of adult and old mice resulted in a two- to four-fold increase in muscle HSP70 content. Treatment of old mice with 17AAG at 3 days prior to and weekly for 4 weeks following a severely damaging contraction protocol resulted in enhanced recovery of force generation at 28 days postdamage compared with muscles of vehicle-treated mice. Data suggest that 17AAG overcomes the mechanism by which activation of the stress response fails in muscles of old mice and may have therapeutic benefit in the recovery following damage in muscles of older individuals.
\end{abstract}

\section{Introduction}

$\mathbf{H}$ EAT SHOCK PROTEINS (HSPS) ACT AS molecular chaperones, facilitating the folding, transportation, and conformational maturation of newly synthesized proteins. ${ }^{1}$ HSPs are also rapidly synthesized following a variety of stresses, including hyperthermia, ischemia, and oxidative stress when they are required for stabilization of denatured or unfolded proteins. This is called the stress response. ${ }^{2,3}$

Increased HSP content has been demonstrated in the skeletal muscle of adult rodents and humans following both acute and long-term exercise protocols. ${ }^{4-7}$ The initiator of this stress response has been proposed to be an increased generation of reactive oxygen species (ROS). ${ }^{8}$ In contrast, the stress response is attenuated in muscles of old rodents following exercise. ${ }^{7,9,10}$ Ageing is associated with an increased susceptibility to contraction-induced damage and a failure to regenerate successfully following exercise-induced damage. ${ }^{11-13}$ This lack of activation of the stress response in muscles of old rodents has been proposed to be a major factor in the development of these age-related functional deficits. ${ }^{12}$

Using transgenic mice, we have previously demonstrated that lifelong overexpression of HSP70 in skeletal muscle has major functional effects on muscles of old mice. ${ }^{12}$ This study demonstrated that overexpression of HSP70 throughout life protected muscles of old mice against the development of secondary damage at 3 days following lengthening contractions and resulted in complete recovery of the ability to generate maximum tetanic force by 28 days following a severely damaging contraction protocol. This complete recovery was similar to muscles of adult wild-type (WT) mice and in contrast to a permanent force deficit at this time point in muscles of old WT mice. ${ }^{11,12}$ These data suggest that the failure of regeneration in muscles of old mice is not inevitable. 
The mechanism by which the stress response is blunted in muscles of old rodents is unclear. The acute synthesis of HSPs in skeletal muscle is controlled by the transcription factor heat shock factor 1 (HSF1), which is expressed constitutively in mature skeletal muscle cells. ${ }^{14}$ In unstressed conditions, HSF1 is present as an inactive monomer and bound to HSP90 and/or HSP70, HSP40, and HSC70 and a complex of other co-chaperones. This association with HSPs is thought to repress HSF1 activation. ${ }^{15,16}$ In response to stress, the appearance of partially unfolded or oxidized cellular proteins triggers the release of HSP70, HSP90, and other chaperones from HSF1 due to the increased affinity for binding of these HSPs for unfolded proteins compared with that for HSF1. ${ }^{15,16}$ HSF1 then trimerizes prior to translocation to the nucleus, where it undergoes further modifications, including phosphorylation to activate transcription of HSP genes. ${ }^{17}$ Previous data suggest that the pattern and extent of ROS generation by muscles of old mice at rest and during contractile activity is modified, ${ }^{18,19}$ and so it may be that the signal for complete activation of HSF1 following exercise is absent in muscles of old rodents. ${ }^{10}$

Several drugs have been developed that increase the cellular content of HSPs. One class of HSP-inducing drugs is a family of HSP90 inhibitors. The HSP90 inhibitors geldanamycin and herbimycin are benzoquinone ansamycin antibiotics and radicicol is a fungal antibiotic. ${ }^{20,21}$ One drawback of geldanamycin is that this drug is hepatotoxic in vivo. ${ }^{22}$ Several synthetic analogues are available, including 17-allylamino-17-demethoxygeldanamycin (17AAG), which is less toxic than gelanamycin but has similar HSP activation properties. ${ }^{23}$ HSP90 inhibitors are thought to activate the stress response by binding to the adenosine triphosphate (ATP)-binding domain of HSP90, preventing binding of HSP90 to HSF1. ${ }^{24}$ Treatment of a variety of cells with HSP90 inhibitors results in increased cellular levels of HSPs, including HSP70, HSP90, HSP40, and HSP25/27. ${ }^{25}$

We hypothesized that treatment of mice with HSP90 inhibitors would result in increased levels of HSPs in skeletal muscle and that this increased HSP content would facilitate complete recovery of force generation by muscles of old mice following damaging contractions in a similar manner to the protection provided by transgenic overexpression of HSP70. ${ }^{12}$

\section{Methods}

\section{Evaluation of the effect of $17 A A G$ on the HSP content of C2C12 myotubes in vitro}

$\mathrm{C} 2 \mathrm{C} 12$ cells were cultured in six-well plates at $37^{\circ} \mathrm{C}$ in $5 \%$ $\mathrm{CO}_{2}$ in Dulbecco modified Eagle medium (DMEM; SigmaAldrich, Dorset, UK) containing 12\% fetal calf serum (FCS). When cells reached 50\% confluence and to induce differentiation, the medium was changed to DMEM containing $2 \%$ horse serum (Invitrogen, UK). At 5 days following addition of $2 \%$ horse serum, cells were treated for $24 \mathrm{~h}$ with $17,1.7$, or $0.17 \mu \mathrm{M}$ of the geldanamycin analogue 17AAG (purchased from Autogen Bioclear, UK) in dimethyl sulfoxide (DMSO) in medium. Control cells were incubated with either medium containing the vehicle DMSO (labeled DMSO) or medium alone (labeled control). Following incubation for $24 \mathrm{~h}$ at $37^{\circ} \mathrm{C}$, cells were harvested in Dulbecco phosphatebuffered saline (D-PBS) and centrifuged for $10 \mathrm{~min}$ at
$20,000 \times g$. The pellets were stored at $-70^{\circ} \mathrm{C}$ for analysis of HSP content.

\section{Mice}

Forty eight adult (12-14 months) and old (24-26 months) male and female C57BL6/J mice were used in this study. Mice were housed in a specific pathogen-free environment with a 12-h light:12-h dark cycle. Experiments were performed in accordance with U.K. Home Office Guidelines under the U.K. Animals (Scientific Procedures) Act 1986, and they received ethical approval from the University of Liverpool Animal Welfare Committee.

\section{Examination of the effect of a single dose of 17AAG on the HSP content of skeletal muscle in vivo}

To examine the effect of a single dose of 17AAG on muscle HSP content, adult and old mice received an intraperitoneal (i.p.) injection of $17 \mathrm{AAG}$ at a dose of $40 \mathrm{mg} / \mathrm{kg}$ body weight in $50 \mu \mathrm{L}$ of DMSO. (The 17AAG for the mouse studies was generously provided by Kosan Biosciences, Inc. and the United States National Cancer Institute, National Institutes of Health.) This dose and route of delivery was chosen because Egorin et al. ${ }^{26}$ had previously demonstrated that this dose resulted in widespread tissue distribution of 17AAG, including skeletal muscle, and when given by the i.p. route resulted in $99 \%$ bioavailability compared with $24 \%$ when given orally. Control mice received i.p. injections of DMSO (vehicle) only or were untreated (no injection). Mice were killed by cervical dislocation at 3 days following treatment, and hind limb muscles were removed and frozen in liquid nitrogen. Extensor digitorum longus (EDL) muscles were analyzed for HSP content as described below.

\section{Time course of muscle HSP content of EDL muscles following 17AAG administration}

Adult mice received a single i.p. injection of $40 \mathrm{mg} / \mathrm{kg}$ of 17AAG and control mice received i.p. vehicle only. Mice were killed on days 1, 2, 3, 6, and 10 following 17AAG administration. EDL muscles were analyzed for HSP content as described below.

\section{Effect of 4 weeks of treatment of with 17AAG on force generation by EDL muscles adult and old mice}

Adult (12-14 months) and old (24-26 months) mice received i.p. injections of $17 \mathrm{AAG}$ at a dose of $40 \mathrm{mg} / \mathrm{kg}$ body weight once each week for 4 weeks. Control mice received vehicle or were untreated. No significant effect of vehicle was observed, and thus control and DMSO data were pooled. Following 4 weeks of treatment with 17AAG, the maximum tetanic force of the EDL muscle was measured in situ using a protocol developed by McCully and Faulkner. ${ }^{27}$ Mice were anesthetized with ketamine hydrochloride (66 $\mathrm{mg} / \mathrm{kg}$ body weight) and medatomidine hydrochloride ( 0.55 $\mathrm{mg} / \mathrm{kg}$ body weight) by i.p. injection. The knee of the right hindlimb was fixed. The distal tendon of the EDL muscle was exposed and attached to the lever arm of a servomotor (Cambridge Technology, USA) but remained intact. The peroneal nerve was exposed, and stainless steel needle electrodes were placed across the nerve. Stimulation voltage (with pulse width of $0.2 \mathrm{mS}$ ) and muscle length were ad- 
justed to produce a maximum twitch force. The optimum muscle length that produced the maximum twitch force is also the optimum muscle length $\left(\mathrm{L}_{0}\right)$ for the production of maximum tetanic force $\left(\mathrm{P}_{0}\right)$. $\mathrm{P}_{0}$ was determined by electrical stimulation of the muscle at $\mathrm{L}_{0}$ for $500 \mathrm{msec}$ at the optimal stimulation voltage (8-10 Volts). The muscle was electrically stimulated every $2 \mathrm{~min}$, and the frequency of stimulation was increased each time from $10 \mathrm{~Hz}$ to $50 \mathrm{~Hz}$ and then in $50-\mathrm{Hz}$ increments to a maximum of $400 \mathrm{~Hz}$. $\mathrm{P}_{0}$ was identified when the maximum force reached a plateau despite increasing stimulation frequency. Mice were killed by cervical dislocation. The weight and length of each EDL muscle was recorded to calculate muscle cross-sectional area (CSA) using 0.44 to correct for fiber length and $1.06 \mathrm{~g} / \mathrm{mL}$ for the density of muscle, ${ }^{28}$ which was then used to calculate specific force. Hind limb muscles were rapidly removed and frozen in liquid nitrogen for later analysis of HSP70 content as described below.

\section{The effect of 17AAG treatment on recovery of force generation following a damaging exercise protocol in old mice}

To determine the effects of 17AAG treatment on the recovery of the EDL muscles of old mice following contraction-induced damage, old (24-26 months) mice received an i.p. injection of $40 \mathrm{mg} / \mathrm{kg}$ body weight $17 \mathrm{AAG}$ or vehicle (DMSO) at 3 days prior to a protocol of 450 damaging lengthening contractions. As described above, mice were anesthetized, and maximum tetanic force generation by the EDL muscles was measured in situ. Following this, the EDL muscles of mice were subjected to a protocol of 450 lengthening contractions at $150 \mathrm{~Hz}$ through a strain of $20 \%$ of $\mathrm{L}_{\mathrm{f}}$ at a velocity of $1.5 \mathrm{~L}_{\mathrm{f}} / \mathrm{s}$, as previously described. ${ }^{12}$ Following this, surgical wounds were sutured, and the mice were allowed to recover. Mice received an i.p. injection of 17AAG or DMSO 7 days following the initial dose (4 days following the contraction protocol) and then every 7 days for 3 weeks. Twenty eight days following the damaging lengthening contraction protocol, and thus at 3 days following the final 17AAG injection, mice were anesthetized and the maximum force of EDL muscles was remeasured. Mice were then killed by cervical dislocation; EDL muscles were excised, weighed, and measured. Both EDL muscles were orientated on cork discs, surrounded by O.C.T. mounting compound, and frozen in iso-pentane precooled in liquid nitrogen. Blocks were stored at $-70^{\circ} \mathrm{C}$ prior to histological analysis of transverse sections. The other hind limb muscles were frozen in liquid nitrogen.

\section{Analysis of muscles for HSP content in cells and skeletal muscle}

Harvested cells were centrifuged at $20,000 \times g$ for $5 \mathrm{~min}$, the supernatant was discarded, and cell pellets were resuspended and sonicated in 1\% sodium dodecyl sulfate (SDS) containing protease inhibitors, as previously described. ${ }^{29}$ Muscles were ground under liquid nitrogen and homogenized 1\% SDS with protease inhibitors and centrifuged at $20,000 \times g$ for $10 \mathrm{~min}$. Protein content was determined using bicinchoninic acid protein assay (Sigma-Aldrich, Dorset, UK). The supernatant was analyzed for HSP content by SDSpolyacrylamide gel electrophoresis (PAGE) and western blotting. Total protein load was standardized by loading 100 $\mu \mathrm{g}$ of total protein into each well of a $7 \times 8-\mathrm{cm} 12 \%$ polyacrylamide gel with a $4 \%$ stacking gel. Proteins were separated at a current of $20 \mathrm{~mA}$ for the stacking gel and $40 \mathrm{~mA}$ for the resolving gel and then transferred onto a nitrocellulose membrane at a current of $0.8 \mathrm{~mA} / \mathrm{cm}^{2}$ for $1.5 \mathrm{~h}$ (Multiphor II discontinuous blotting system, Pharmacia, Milton Keynes, UK). The membrane was probed with antibodies for HSP25 (Stressgen, Victoria SPA-801), HSP70 (Stressgen, Victoria SPA-810), HSC70 (Stressgen, Victoria SPA-815), and HSP60 (Sigma-Aldrich, H3524). Recombinant HSP proteins (Stressgen, Victoria, Canada) were run alongside samples as positive controls on each western blot. Western blots were shown using an enhanced chemiluminescence (ECL) detection system (Perbio Science, Northumberland, UK) with ChemidocXRS (Bio-Rad, Hercules, USA) and analyzed by densitometry using QuantityOne software (Bio-Rad, Hercules, USA). Graphical data from western blots are presented as a percentage of the mean adult control value. Statistical analysis was carried out on raw densitometric data.

\section{Histological analysis}

Ten-micron transverse sections were cut through the midpoint of the EDL muscle using a cryostat (Leica CM1850, Leica Microsystems, Germany), transferred onto glass slides, and stained with Hematoxylin \& Eosin (Merck Ltd, Dorset UK), as previously described. ${ }^{12}$ EDL muscles from both DMSO- and 17AAG-treated mice were examined at 28 days following the damaging lengthening contraction protocol. EDL muscles that had undergone the contraction protocol were compared with the contralateral undamaged EDL. Regenerating fibers were identified by the presence of centrally positioned nuclei. The total number of fibers and the number of regenerating fibers were counted for three full sections of the damaged EDL for each mouse. These data were averaged per muscle, and the number of regenerating fibers was expressed as a percentage of the total number of fibers. The mean number of fibers per section was 234 and a mean of 702 total fibers were counted per mouse.

\section{Statistical analysis}

Statistical analysis was carried out using Statistical Package for Social Sciences (SPSS) software Version 13. Student $t$-tests were carried out to analyze differences between two groups. Where multiple comparisons were made, data were analyzed using analysis of variance (ANOVA) accounting for repeated measures where necessary. When a significant F value was observed, Tukey's post hoc analysis was performed to identify where differences occurred. Significance was set at the alpha level of $\leq 0.05$. Data are presented as mean \pm the standard error of the mean (SEM).

\section{Results}

\section{Treatment of C2C12 myotubes with 17AAG resulted in increased content of HSPS}

The HSP70, HSC70, and HSP25 contents of C2C12 cells were increased following treatment with 17AAG compared with cells treated with DMSO (vehicle) or untreated cells (Fig. 1). No significant dose-response was observed in the induction of HSP70, with all concentrations of 17AAG used resulting in $\sim 10$-fold increase in HSP70 compared with un- 
17AAG Treated

\begin{tabular}{|c|c|c|c|c|c|c|c|c|}
\hline $17 \mu \mathrm{M}$ & $1.7 \mu \mathrm{M}$ & $0.17 \mu \mathrm{M}$ & \multicolumn{2}{|c|}{ DMSO } & \multicolumn{2}{|c|}{ Control } & $\begin{array}{l}\text { positive } \\
\text { control }\end{array}$ & \multirow[b]{2}{*}{ HSP70 } \\
\hline 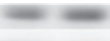 & $=0$ & $=10$ & & & 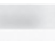 & & $=$ & \\
\hline $\mathbf{0}$ & $=$ & 00 & 10 & $\mathbf{m}$ & 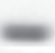 & 10 & $=5$ & HSC70 \\
\hline & & $2 \mathrm{~m}$ & 28 & $a=$ & 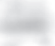 & 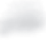 & $10=8$ & HSP25 \\
\hline & & & & $=$ & $=$ & 10 & & \\
\hline
\end{tabular}

FIG. 1. Western blots showing the HSP70, HSC70, HSP25, and HSP60 content of untreated C2C12 cells and cells following $24 \mathrm{~h}$ of treatment with DMSO or 17AAG at 17, 1.7, and $0.17 \mu \mathrm{M}$.

treated control cells. HSC70 was increased in C2C12 cells by approximately $50 \%$ following treatment with the highest concentration of 17AAG compared with untreated control cells. The lower concentrations resulted in $20-30 \%$ increases in HSC70 compared with control untreated cells. DMSO treatment alone resulted in an increase in the HSP25 content of $\mathrm{C} 2 \mathrm{C} 12$ cells to $\sim 200 \%$ of the content of untreated cells. HSP25 was further increased to $\sim 275 \%$ of control cells following treatment with $0.17 \mu \mathrm{M}$ 17AAG in DMSO and $400 \%$ with $17 \mu \mathrm{M}$ 17AAG in DMSO. An $\sim 70 \%$ increase in the HSP60 content of C2C12 cells was observed following treatment with either DMSO or 0.17 $\mu \mathrm{M}$ 17AAG in DMSO.

\section{A single dose of 17AAG resulted in a significant increase in the HSP70 content of skeletal muscle of adult mice in vivo}

Treatment of adult mice with a dose of $40 \mathrm{mg} / \mathrm{kg}$ body weight of 17AAG resulted in a significant increase in the HSP70 content of EDL muscles compared with DMSO vehicle and untreated control mice at 3 days following the injection (17AAG, $218.7 \pm 29.2 \%$ of control mice; DMSO, $73.3 \pm$ $6.2 \%$ of control; Fig. 2a,b). In contrast, no effect of 17AAG was seen on the HSC70 and HSP25 content of EDL muscles compared with muscles of DMSO-treated and untreated mice (Fig. 2a,b).

\section{HSP70 content of EDL muscles remained elevated for 10 days following 17AAG administration}

The HSP70 content of EDL muscles of adult mice was elevated at $24 \mathrm{~h}$ following treatment with a single i.p. dose of $40 \mathrm{mg} / \mathrm{kg}$ body weight of $17 \mathrm{AAG}$ and remained elevated at 10 days following treatment compared with muscles of control mice (Fig. 2c).

\section{Effects of 4 weeks of 17AAG treatment on the HSP7O content of quadriceps muscle and contractile characteristics of EDL muscles}

Western blot analysis confirmed that HSP70 was elevated in the skeletal muscle of adult and old mice following a single i.p. injection of 17AAG every 7 days for 4 weeks. Data demonstrated that 4 weeks of treatment with 17AAG resulted in a four-fold increase in the HSP70 content of quadriceps muscles of adult mice compared with muscles of adult control mice. In muscles of old mice, 4 weeks of treatment with 17AAG resulted in an increase in the HSP70 content of quadriceps muscles to a similar level to that of adult 17AAGtreated mice. Old control mice tended to have higher HSP70 content than adult control mice such that the resulting increase following 17AAG treatment was two-fold compared with age-matched control mice (Fig. 3).

Table 1 shows the contractile properties of EDL muscles of adult and old mice following 4 weeks of treatment with 17AAG. Muscles of old 17AAG-treated and old control mice had a significantly reduced maximum tetanic force compared with muscles of adult control mice (Fig. 4a). Maximum specific force was not significantly different between any groups studied (Fig. 4b). Four weeks of 17AAG treatment did not result in any significant changes in muscle mass, CSA, or in any of the twitch characteristics measured in adult

$\mathbf{a}$

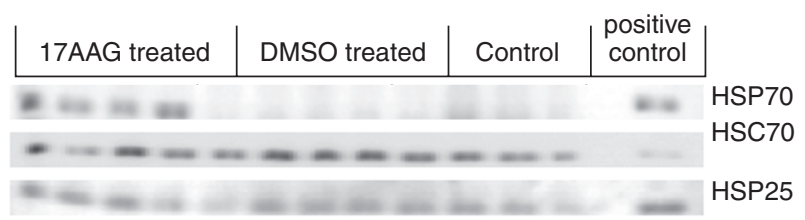

b

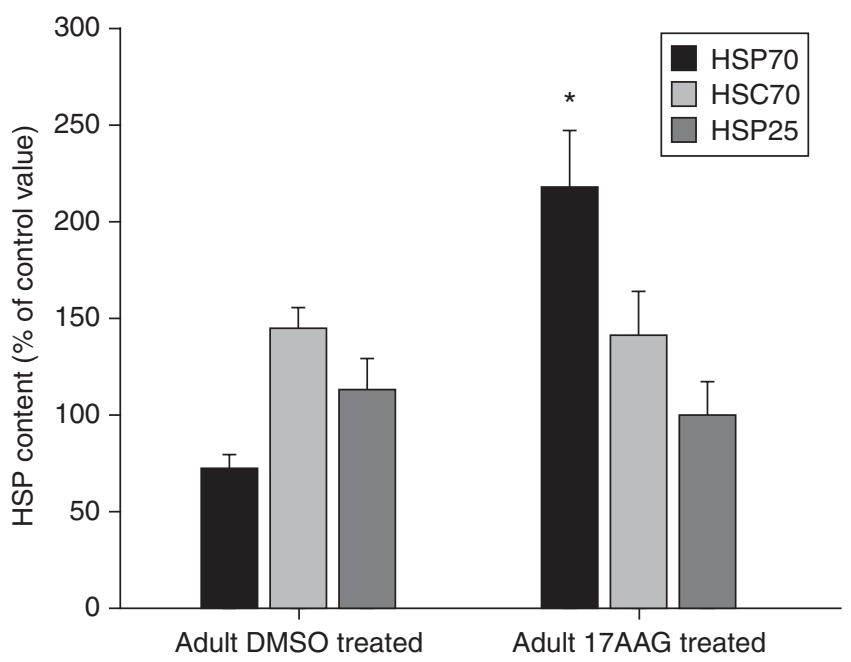

c

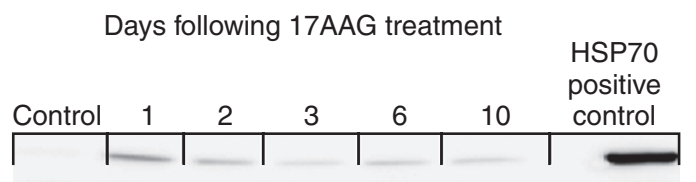

FIG. 2. (a) Western blots showing HSP70, HSC70, and HSP2 5 content of EDL muscles of untreated control mice and mice at 3 days following treatment with a single dose of 17AAG or DMSO. (b) HSP70, HSC70, and HSP25 content of EDL muscles of DMSO-treated and 17AAG-treated mice as a percentage of the content in muscles of control untreated mice at 3 days following a single dose $\left(^{*}\right) p<0.05$ compared with values for adult DMSO treated mice $(n=4)$. (c) Western blot showing HSP70 content of EDL muscles of untreated control mice and mice on days $1,2,3,6$, and 10 days following a single dose of 17AAG. 


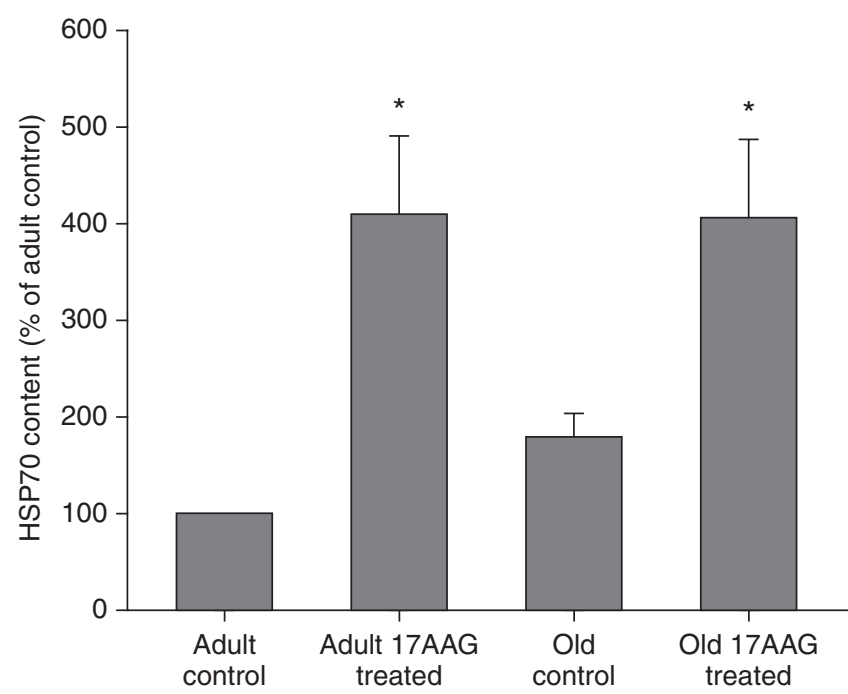

FIG. 3. HSP70 content of quadriceps muscles of adult and old control and 17AAG-treated mice following 4 weeks of treatment. $\left(^{*}\right) p<0.05$ compared with values for adult control treated mice $(n=3-4)$.

mice. In addition, 4 weeks of 17AAG treatment did not result in any significant differences in maximum tetanic or specific force. Time-to-peak twitch and half-relaxation time were significantly greater for muscles of old 17AAG and control mice compared with adult control mice, thus this effect appeared to be due to age rather than 17AAG treatment. No significant differences were observed between the contractile characteristics of EDL muscles of old 17AAG-treated and old control mice apart from CSA, which was significantly lower in 17AAG-treated mice compared with control mice.

\section{Treatment of old mice with 17AAG treatment enhances recovery of $E D L$ muscles following lengthening contraction-induced damage}

Twenty eight days following the protocol of 450 lengthening contractions, the maximum tetanic force generation by EDL muscles of 17AAG-treated old mice had recovered to $83.6 \pm 9.6 \%$ of the predamage maximum tetanic force that was not significantly different from the initial (predamage) tetanic force values. In contrast, maximum tetanic force generation of EDL muscles of DMSO-treated mice had only recovered to $48.3 \pm 8.2 \%$ of the predamage maximum tetanic force, a significant reduction compared with the initial (predamage) maximum tetanic force value (Fig. 5). Thus, there was a statistically significant improvement in recovery of maximum tetanic force in 17AAG-treated old mice compared with DMSO-treated old mice. Histological analysis of transverse muscle sections at 28 days following the damaging lengthening contraction protocol demonstrated that $39.3 \pm 11.7 \%$ of fibers in DMSO-treated mice had centrally positioned nuclei, indicative of regenerating muscle fibres, compared with $22.5 \pm 5.7 \%$ in 17 AAG mice (Fig. 6). These values were not significantly different.

\section{Discussion}

This study investigated whether treatment of mice with the known HSP inducer 17AAG resulted in increased HSP70 content of skeletal muscle and whether this would result in improved recovery of force generation in muscles of old mice following lengthening contraction-induced damage, in a similar manner to the improved recovery following contraction-induced damage seen in muscles of old HSP70 overexpressor mice. ${ }^{12}$

Data presented demonstrate that $17 \mathrm{AAG}$ increased the HSP70, HSP25, and HSC70 content of muscle cells in vitro and the HSP70 content of skeletal muscle in vivo (Figs. 1 and 2) and this increased content was maintained for up to 6 days posttreatment. Data demonstrated that 4 weeks of 17AAG treatment in vivo resulted in increased content of HSP70 in muscles of adult and old mice (Fig. 3). This induction by 17AAG of HSP production in muscles of old mice is in contrast to the attenuated production of HSPs by muscles of old mice following exercise $^{7}$ and suggests that there is no intrinsic failure of HSF1 function in muscles of old mice. The different pattern of HSP induction in muscle cells in culture compared with muscle of mice in vivo following treatment with 17AAG may be due to differences in the dose and time that the muscle cells were exposed to in each situation. Although we did not examine the half-life of 17AAG in our cell culture studies or the delivery of 17AAG to muscles in vivo, other workers have demonstrated that, when given by the i.p. route, $40 \mathrm{mg} / \mathrm{kg}$ of $17 \mathrm{AAG}$ resulted in peak plasma concentrations at $1 \mathrm{~h}$ postdelivery, which declined to below detectable levels at $4 \mathrm{~h}$, resulting in $99 \%$ bioavailability in mice and widespread tissue distribution of 17AAG, including skeletal muscle. ${ }^{26}$ We would speculate that the cells in culture would be exposed to the 17AAG for longer periods than $4 \mathrm{~h}$, and this may explain the greater stress response in the muscle cells in culture.

Table 1. Contractile Characteristics of EDl Muscles of Adult and Old Control and 17AAG-Treated Mice

\begin{tabular}{lcccc}
\hline & Adult control & $\begin{array}{c}\text { Adult 17AAG } \\
\text { treated }\end{array}$ & Old control & $\begin{array}{c}\text { Old 17AAG } \\
\text { treated }\end{array}$ \\
\hline Muscle mass (mg) & $12.8 \pm 0.4$ & $11.8 \pm 0.6$ & $12.1 \pm 0.6$ & $10.0 \pm 2.0$ \\
Muscle CSA (mm $\left.{ }^{2}\right)$ & $2.8 \pm 0.1$ & $2.6 \pm 0.1$ & $2.5 \pm 0.1$ & $2.3 \pm 0.2^{\mathrm{a}}$ \\
$\begin{array}{l}\text { Peak twitch force }(\mathrm{mN}) \\
\begin{array}{l}\text { Time to preak twitch } \\
\text { (msec) }\end{array}\end{array}$ & $141 \pm 14$ & $151 \pm 28$ & $119 \pm 9$ & $116 \pm 27$ \\
$\begin{array}{l}\text { Twitch half relaxation } \\
\text { time (msec) }\end{array}$ & $8.7 \pm 0.4$ & $8.8 \pm 0.7$ & $11.6 \pm 0.7^{\mathrm{a}}$ & $10.5 \pm 1.2^{\mathrm{a}}$ \\
\hline
\end{tabular}

a $p<0.05$ compared with adult control value. Values are means \pm SEM.

EDL, Extensor digitorum longus; AAG, 17-(allylamino)-17-demethoxygeldanamycin. 
$\mathbf{a}$

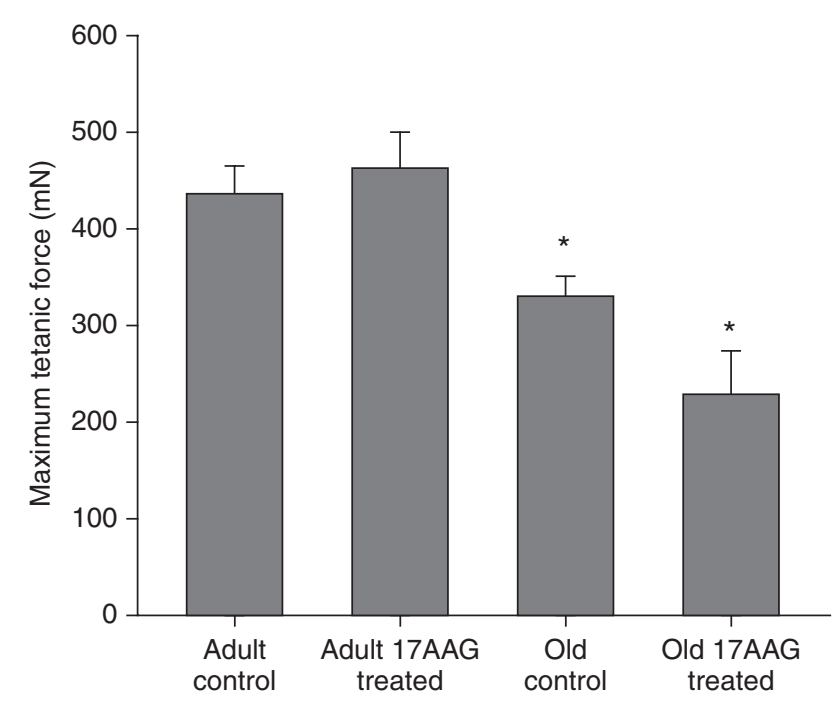

b

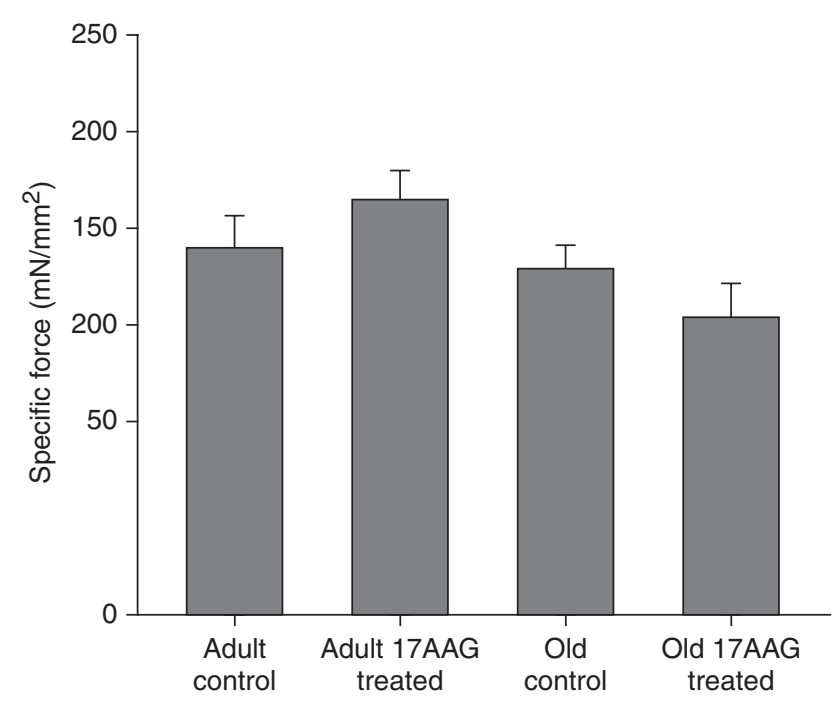

FIG. 4. Maximum tetanic (a) and specific (b) force generated by EDL muscles of adult and old control and 17AAGtreated mice following 4 weeks of treatment. $\left.{ }^{*}\right) p<0.05$ compared with adult control value. Values are means \pm SEM.

In the in vivo damage and regeneration study control mice received an equal volume of DMSO as vehicle to 17AAGtreated mice to rule out an effect of the DMSO vehicle on regeneration. Reid and Moody ${ }^{30}$ demonstrated in isolated fiber bundles from rat diaphragm that DMSO decreased force generation at low frequency $(30-60 \mathrm{~Hz})$ but did not affect force at maximum tetanus $(200 \mathrm{~Hz})$; however, this is unlikely to have affected force in the muscles of mice in the present study because DMSO or 17AAG was administered 3 days prior to the determination of maximum force generation. In addition, a pharmacokinetic study in mice by Kaye et al. ${ }^{31}$ demonstrated that, at $8 \mathrm{~h}$ following an intravenous (i.v.) dose of DMSO of $1.5 \mathrm{mg} / \mathrm{g}$ body weight, there was little or no detectable DMSO in plasma or tissue. In the current study,
DMSO was administered i.p. and maximum force generation was measured 3 days later, suggesting that no effect of DMSO on force generation would be observed.

The increased muscle content of HSP70 in old mice induced by treatment with 17AAG both 3 days before damage and for 4 weeks during recovery following a severe lengthening contraction protocol was associated with a significant improvement in recovery of force generation at 28 days postdamage in EDL muscles of 17AAG-treated mice compared with vehicle-treated mice (Fig. 5). Maximum force generated by EDL muscles of 17AAG-treated mice had returned to predamage maximum force values by 28 days following the damaging contraction protocol. In contrast, muscles of vehicle (DMSO)-treated mice recovered to $\sim 48.3 \%$ of the predamage force value. These data are comparable to that of McArdle et al., ${ }^{12}$ which showed that muscles of old WT mice had a $44 \%$ maximum force deficit at 28 days following the same damaging contraction protocol. In contrast, no effect of 4 weeks of 17AAG treatment was seen on maximum force generation in these mice; thus, the $\mathrm{P}_{\mathrm{o}}$ of old 17AAG-treated mice remained significantly less than that of adult control mice after 4 weeks of treatment. A large proportion of the age-related loss of maximum force generation occurs as a result of muscle fiber loss, and it would be unlikely that such an acute treatment with AAG would affect this loss. However, lifelong HSP70 overexpression also preserved regeneration without protecting against age-related loss of maximum tetanic force generation.

It has been hypothesized that the age-related lack of complete regeneration in muscles is one of the major causes of muscle weakness; thus, correction of this failure of regeneration may have a substantial effect on muscle function. ${ }^{32}$ The reduction in maximum tetanic force generation is largely due to a loss of muscle fibers, ${ }^{11}$ and this occurs over a much greater time period than the 4 -week treatment period. Thus, substantial loss is likely to have already occurred to a great

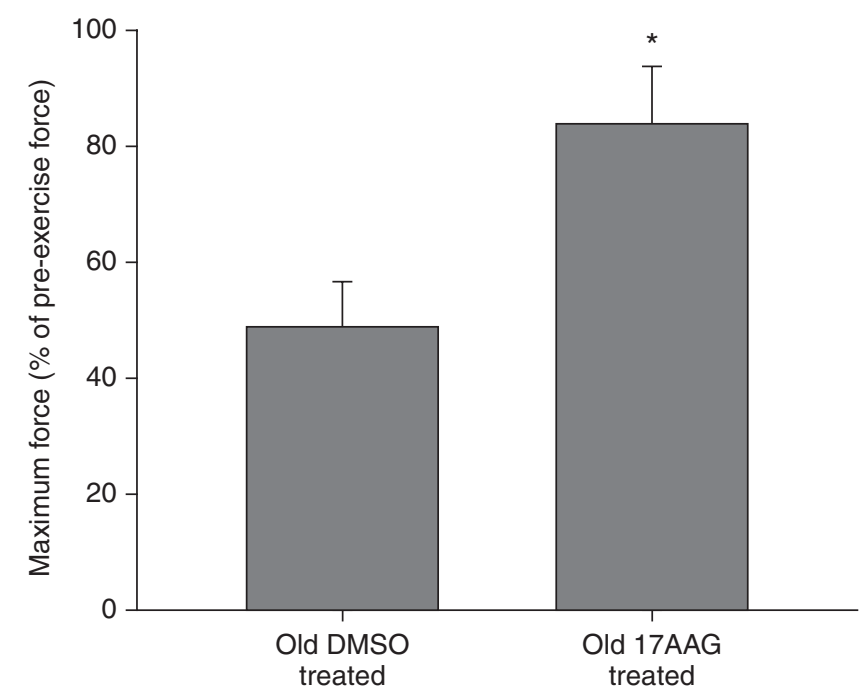

FIG. 5. Maximum tetanic force generated by EDL muscles of old DMSO and 17AAG-treated mice at 28 days following the lengthening contraction protocol expressed as a percentage of initial predamage force. $\left(^{*}\right) p<0.05$ compared with the value for DMSO-treated mice $(n=4-5)$. 

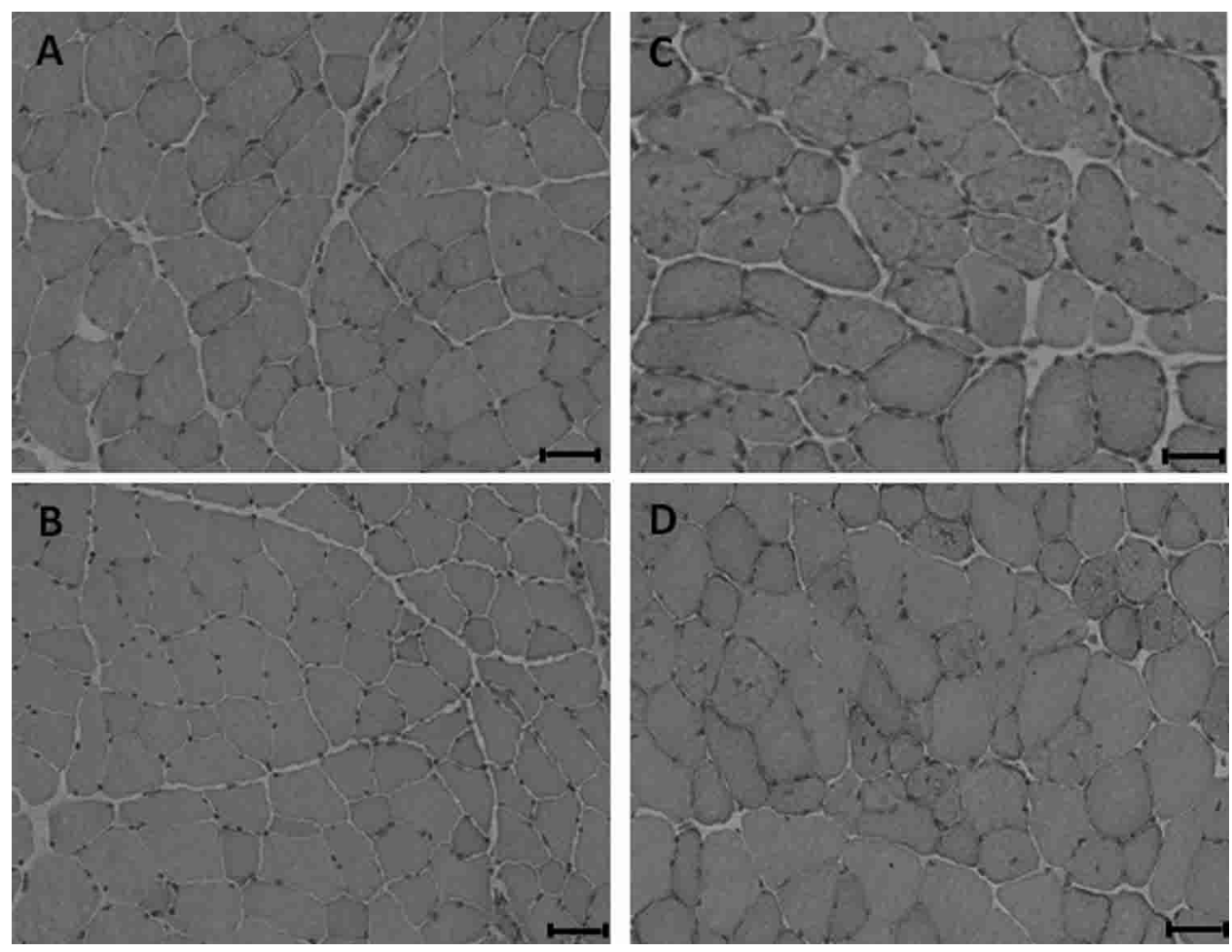

FIG. 6. Representative transverse sections of EDL muscles of old DMSO and 17AAG treated mice. (A) Undamaged contralateral EDL muscle of old DMSO-treated mouse. (B) Undamaged contralateral EDL muscle of old 17AAG-treated mouse. (C) EDL muscle of old DMSO-treated mouse at 28 days following lengthening contractions. (D) EDL muscle of old 17AAGtreated mouse at 28 days following lengthening contractions. Scale bar, $20 \mu \mathrm{m}$.

extent prior to the start of this acute treatment. However, studies in transgenic mice overexpressing HSP70 throughout life do not support the hypothesis that longer-term increases in muscle content of HSP70 would protect against this age-related loss of tetanic force generation, ${ }^{12}$ suggesting that failed ability to regenerate successfully does not play a major role in the loss of maximum tetanic force generation. Evidence that increased HSP70 content of EDL muscles facilitates successful regeneration following damage in old mice is consistent with that obtained using transgenic mice overexpressing HSP70 in skeletal muscle ${ }^{12}$ and suggests that relatively acute induction of increased HSP70 content of muscles provides similar protection to that achieved by lifelong increased muscle content of HSP70. The effects of shortand lon c $\mathrm{m}$ increases in the HSP70 content of muscles in correcti he failed regeneration in muscles of old mice without prevention of age-related atrophy in muscles of old mice suggest that the presence of functional HSP70 and/or associated protection against accumulation of markers of oxidation and preservation of nuclear factor $-\kappa \mathrm{B}(\mathrm{NF}-\kappa \mathrm{B})$ responses ${ }^{14}$ may be necessary for successful regeneration, but not for protection against the development of atrophy. These data do not however rule out the possibility that other HSPs may be involved in preservation of muscle against the development of age-related atrophy.

The levels of HSP70 that facilitate successful regeneration suggest that there may be a minimum induction level at which HSP70 provides this protection. In the transgenic study, the level of overexpression of HSP70 in heterozygotic and homozygotic mice was between 10- and 20-fold that of muscles of WT mice. In the present study, it was not possible to measure the HSP content of EDL muscles, but treatment with 17AAG for 4 weeks resulted in a four-fold increase in the HSP70 content of quadriceps muscles of adult mice (Fig. 3). The EDL muscle is comprised of primarily of type 2 muscle fibers and shows an age-related reduction in mass and force generation. Therefore, it is appropriate for studying the mechanisms of skeletal muscle loss with ageing. ${ }^{11,12}$ In the current study, this muscle was also used for histological examination for evidence of regenerating muscle fibers following contraction-induced damage in comparison with undamaged contralateral muscles and therefore was not available for analysis of HSP content. However, as discussed in our previous study, ${ }^{4}$ the quadriceps and EDL are primarily comprised of the same fiber type in the mouse and we would therefore expect 17AAG to elicit similar effects in both muscles. The fold induction of HSP70 was lower in the quadriceps muscles of old 17AAG-treated mice, but this was due to higher basal levels of HSP70 in muscles of old mice in general prior to treatment, ${ }^{4}$ and so the absolute levels of HSP70 achieved in muscles of adult and old mice were similar.

Previously, we demonstrated an increase in basal HSP70 content in muscles of old mice compared with adult mice; however, it is unclear whether these higher basal levels are protective or may be a result of reduced proteasomal activity resulting in accumulation of oxidized proteins in muscles of old mice. ${ }^{4}$ Data presented suggest that a level of induc- 
tion of HSP70 in muscle of greater than two-fold of normal levels results in full recovery of force generation in muscles of old mice following lengthening contractions. This suggests that there may be a threshold level at which HSP70 enhances recovery in muscle.

Other data from our laboratory have demonstrated that the physiological stress of a mild treadmill training protocol in mice for 12 months, from adult to old age, resulted in a $<1.5$-fold increase in the HSP70 content of skeletal muscle at old age, and muscles of these mice did not demonstrate significantly improved recovery from a severe lengthening contraction protocol of 450 contractions. ${ }^{4}$ This suggests that the increase in HSP70 required for improved recovery of muscles of old mice following severe damage is likely to be greater than two-fold.

The mechanism by which increased muscle content of HSP70 improved regeneration following damaging lengthening contractions is unclear. Previous work from our laboratory has demonstrated that markers of oxidative stress are elevated in the muscle of old mice, including increases in catalase and superoxide dismutase activities, increased in lipid peroxidation (malonaldehyde), and decreased glutathione content. ${ }^{14}$ HSP70 overexpression preserved the ability of muscles of old mice to activate the redox-sensitive transcription factor NF- $\kappa$ B following a period of nondamaging isometric exercise. It has been demonstrated that NF- $\kappa \mathrm{B}$ activation is essential during differentiation of myoblasts to form myotubes and during maturation of muscles during regeneration, and that this process is aberrant in muscles of old mice. ${ }^{33}$ We hypothesize that the preservation of muscle function in old HSP70 overexpressor mice may be, at least in part, a result of maintenance of the ability to activate NF$\kappa \mathrm{B}$-mediated transcription following stress although the effect of 17AAG treatment on NF- $\kappa$ B activation was not determined in the current study.

The data presented here demonstrated that 17AAGtreated mice had significantly improved recovery of force generation following damage and no statistical difference in the number of regenerating fibers at 28 days postdamage (Figs. 5 and 6). We propose that this improvement in force generation is due to enhanced regeneration as a result of elevated HSP70 content of muscles rather than reduced initial damage. Although we did not examine initial force loss at 3 $\mathrm{h}$ postinjury in 17AAG-treated mice, data from a previous study ${ }^{12}$ demonstrate that HSP70 overexpression did not protect against the initial injury caused by 450 lengthening contractions and resulted in similar force losses in WT and HSP70 overexpressor mice. Therefore, we would not expect any protection in muscle of 17AAG-treated mice at this time point; however, because initial damage was not assessed, it is not possible to exclude reduced initial damage as the basis of the improved regeneration in muscles of old 17AAGtreated mice compared with old vehicle (DMSO)-treated mice. In addition, McArdle et al. ${ }^{12}$ demonstrated that muscles of HSP70 overexpressor mice were protected against the secondary loss of force at 3 days postinjury, which is hypothesized to be a result of widespread necrosis and secondary free radical-mediated injury as a result of invading phagocytic cells. ${ }^{13}$ It is possible that the successful regeneration of muscles of 17AAG-treated mice was a result of protection against this secondary loss of force, although previous data demonstrated that there is no intrinsic failure in force generation in muscles of old mice until the later stages of maturation seen at 14 days following this contraction protocol. ${ }^{12}$

The mechanisms by which recovery of force generation fails in old mice at this relatively late time point are unclear. Several aspects of maturation occur at later time points following damage, including the expression of maturation-related genes such as adult myosins, ${ }^{34}$ and maturation is, at least in part, controlled by innervations. ${ }^{35}$ The lack of statistical difference in the number of regenerating fibers in muscles of vehicle versus 17AAG-treated mice is intriguing. The timing and reason for muscle nuclei to remain central during regeneration is very poorly understood. Previous data from HSP70 overexpressor mice has demonstrated a more rapid regeneration when determined by measurement of maximum force generation in muscles with increased HSP70 content, and so it is possible that, by 28 days following damage, the nuclei have returned to the periphery in some muscle fibers, particularly those treated with 17AAG.

Because 17AAG was given by i.p. injection in the current study, this may result in increased HSP70 in many tissues. ${ }^{26}$ Therefore, it is not possible to exclude the effects of increased HSP70 content in other tissues, including motor neurons, on muscle regeneration following contraction-induced damage. Furthermore, additional cellular functions of HSP90 inhibitors cannot be ruled out as playing a role in the mechanism by which regeneration is facilitated in the current study. Thus, in addition to activating transcription of HSPs, 17AAG inhibits the chaperone function of HSP90 by binding to the conserved ATP binding domain in the amino-terminal region, preventing the formation of the mature HSP90-client protein complex and therefore preventing ATP-dependent chaperone activity of HSP90. As a result, HSP90 client proteins (which include steroid hormone receptors, cell cycle regulators such as cdk4, transcription factors such as p53, and enzymes such as nitric oxide syntheses) do not achieve their functional conformation. This can lead to the degradation of these client proteins by the ubiquitin-proteasome pathway. ${ }^{25}$ Therefore, although the positive effects of 17AAG are associated with increased muscle content of HSP70, the effects of 17AAG on regeneration of muscle cannot solely be attributed to the increase in HSP70 in muscle without further investigation.

Increased HSP content of muscles has been shown to be cytoprotective in various circumstances. ${ }^{12,29,36,37}$. Pharmacological induction of HSP70 has previously been demonstrated to protect muscles of mice following noncontractioninduced damage. Miyabara et al. ${ }^{38}$ demonstrated reduced muscle damage following cryolesion in muscles of transgenic HSP70 overexpresser mice and in muscles of mice treated with the HSP90 inhibitor radicicol. This study also demonstrated that mice overexpressing HSP70 had fewer regenerating fibers and satellite cells at 10 days postinjury, although the authors could not determine whether this was a result of reduced initial damage or improved regeneration. Because the radicicol was only administered immediately following a cryolesion, it is likely that the increased HSP70 content affected regeneration rather than initial damage.

\section{Perspectives and Significance}

In summary, this is the first study to demonstrate complete recovery of force generation in muscles of old WT mice 
following lengthening contraction-induced injury by pharmacological elevation of HSP70. These data provide evidence that pharmacological 17AAG treatment enhances recovery of muscle of old WT mice following severe contraction-induced damage. The use of inducers of HSPs such as 17AAG as therapeutic agents may have wide-ranging applications in treatment of skeletal muscle injury in young and old individuals; however, further studies are required to elucidate the mechanism by which increased cellular content of HSP70 enhances recovery following contraction-induced damage.

\section{Acknowledgments}

The authors would like to thank Research into Ageing for generous financial support. The 17AAG for the mouse studies was generously provided by Kosan Biosciences, Inc. and the National Cancer Institute, National Institutes of Health.

\section{References}

1. Locke M, Noble EG. Stress proteins: the exercise response. Can J Appl Physiol 1995;20:155-167.

2. Kiang JG, Tsokos GC. Heat shock protein $70 \mathrm{kDa}$ : molecular biology, biochemistry, and physiology. Pharmacol Ther 1998;80:183-201.

3. Liu Y, Steinacker JM. Changes in skeletal muscle heat shock proteins: pathological significance. Front Biosci 2001;6: D12-D25.

4. Kayani AC, Close GL, Jackson MJ, McArdle A. Prolonged treadmill training increases HSP70 in skeletal muscle but does not affect age-related functional deficits. Am J Physiol Regul Integr Comp Physiol 2008;294:R568-R576.

5. McArdle A, Pattwell D, Vasilaki A, et al. Contractile activity-induced oxidative stress: cellular origin and adaptive responses. Am J Physiol Cell Physiol 2001;280:C621C627.

6. Morton JP, MacLaren DP, Cable NT, et al. Time course and differential responses of the major heat shock protein families in human skeletal muscle following acute nondamaging treadmill exercise. J Appl Physiol 2006;101:176-182.

7. Vasilaki A, Jackson MJ, McArdle A. Attenuated HSP70 response in skeletal muscle of aged rats following contractile activity. Muscle Nerve 2002;25:902-905.

8. McArdle A, Vasilaki A, Jackson M. Exercise and skeletal muscle ageing: cellular and molecular mechanisms. Ageing Res Rev 2002;1:79-93.

9. Naito H, Powers SK, Demirel HA, Aoki J. Exercise training increases heat shock protein in skeletal muscles of old rats. Med Sci Sports Exerc 2001;33:729-734.

10. Vasilaki A, McArdle F, Iwanejko LM, McArdle A. Adaptive responses of mouse skeletal muscle to contractile activity: The effect of age. Mech Ageing Dev 2006;127: 830-839.

11. Brooks SV, Faulkner JA. Contraction-induced injury: recovery of skeletal muscles in young and old mice. Am J Physiol 1990;258:C436-C442.

12. McArdle A, Dillmann WH, Mestril R, et al. Overexpression of HSP70 in mouse skeletal muscle protects against muscle damage and age-related muscle dysfunction. FASEB J 2004;18:355-357.

13. Zerba E, Komorowski TE, Faulkner JA. Free radical injury to skeletal muscles of young, adult, and old mice. Am J Physiol 1990;258:C429-435.
14. Broome CS, Kayani AC, Palomero J, et al. Effect of lifelong overexpression of HSP70 in skeletal muscle on age-related oxidative stress and adaptation after nondamaging contractile activity. FASEB J 2006;20:1549-1551.

15. Shi Y, Mosser DD, Morimoto RI. Molecular chaperones as HSF1-specific transcriptional repressors. Genes Dev 1998;12: 654-666.

16. Zou J, Guo Y, Guettouche T, et al. Repression of heat shock transcription factor HSF1 activation by HSP90 (HSP90 complex) that forms a stress-sensitive complex with HSF1. Cell 1998;94:471-480.

17. Santoro MG. Heat shock factors and the control of the stress response. Biochem Pharmacol 2000;59:55-63.

18. Close GL, Kayani AC, Ashton T, et al. Release of superoxide from skeletal muscle of adult and old mice: an experimental test of the reductive hotspot hypothesis. Aging Cell 2007;6:189-195.

19. Vasilaki A, Mansouri A, Remmen $\mathrm{H}$, et al. Free radical generation by skeletal muscle of adult and old mice: effect of contractile activity. Aging Cell 2006;5:109-117.

20. Chiosis G, Vilenchik M, Kim J, Solit D. Hsp90: the vulnerable chaperone. Drug Discov Today 2004;9:881-888.

21. Westerheide SD, Morimoto RI. Heat shock response modulators as therapeutic tools for diseases of protein conformation. J Biol Chem 2005;280:33097-33100.

22. Behrsing HP, Amin K, Ip C, et al. In vitro detection of differential and cell-specific hepatobiliary toxicity induced by geldanamycin and 17-allylaminogeldanamycin in rats. Toxicol In Vitro 2005;19:1079-1088.

23. Schulte TW, Neckers LM. The benzoquinone ansamycin 17allylamino-17-demethoxygeldanamycin binds to HSP90 and shares important biologic activities with geldanamycin. Cancer Chemother Pharmacol 1998;42:273-279.

24. Whitesell L, Bagatell R, Falsey R. The stress response: implications for the clinical development of hsp90 inhibitors. Curr Cancer Drug Targets 2003;3:349-358.

25. Kamal A, Boehm MF, Burrows FJ. Therapeutic and diagnostic implications of Hsp90 activation. Trends Mol Med 2004;10:283-290.

26. Egorin MJ, Zuhowski EG, Rosen DM, et al. Plasma pharmacokinetics and tissue distribution of 17-(allylamino)-17demethoxygeldanamycin (NSC 330507) in CD2F1 mice1. Cancer Chemother Pharmacol 2001;47:291-302.

27. McCully KK, Faulkner JA. Injury to skeletal muscle fibers of mice following lengthening contractions. J Appl Physiol 1985;59:119-126.

28. McCully KK, Faulkner JA. Characteristics of lengthening contractions associated with injury to skeletal muscle fibers. J Appl Physiol 1986;61:293-299.

29. Maglara AA, Vasilaki A, Jackson MJ, McArdle A. Damage to developing mouse skeletal muscle myotubes in culture: protective effect of heat shock proteins. J Physiol 2003;548(Pt 3):837-846.

30. Reid MB, Moody MR. Dimethyl sulfoxide depresses skeletal muscle contractility. J Appl Physiol 1994;76:2186-2190.

31. Kaye TS, Egorin MJ, Riggs CE, Jr., et al. The plasma pharmacokinetics and tissue distribution of dimethyl sulfoxide in mice. Life Sci 1983;33:1223-1230.

32. Faulkner JA, Brooks SV, Zerba E. Muscle atrophy and weakness with aging: contraction-induced injury as an underlying mechanism. J Gerontol A Biol Sci Med Sci 1995;50(Spec No):124-129.

33. Guttridge DC, Albanese C, Reuther JY, et al. NF-kappaB controls cell growth and differentiation through transcriptional regulation of cyclin D1. Mol Cell Biol 1999;19:5785-5799. 
34. Goetsch SC, Hawke TJ, Gallardo TD, et al. Transcriptional profiling and regulation of the extracellular matrix during muscle regeneration. Physiol Genomics 2003;14:261-271.

35. Kobayashi T, Askanas V, Engel WK. Human muscle cultured in monolayer and cocultured with fetal rat spinal cord: importance of dorsal root ganglia for achieving successful functional innervation. J Neurosci 1987;7:3131-3141.

36. Selsby JT, Dodd SL. Heat treatment reduces oxidative stress and protects muscle mass during immobilization. Am J Physiol Regul Integr Comp Physiol 2005;289:R134-R139.

37. Selsby JT, Rother S, Tsuda S, et al. Intermittent hyperthermia enhances skeletal muscle regrowth and attenuates oxidative damage following reloading. J Appl Physiol 2007; 102:1702-1707.

38. Miyabara EH, Martin JL, Griffin TM, et al. Overexpression of inducible $70-\mathrm{kDa}$ heat shock protein in mouse attenuates skeletal muscle damage induced by cryolesioning. Am J Physiol Cell Physiol 2006;290:C1128-C1138.
Address reprint requests to: Professor A. McArdle School of Clinical Sciences University of Liverpool Liverpool CH47 $3 A G$ United Kingdom

Received: September 2, 2008 Accepted: October 30, 2008

E-mail: mdcr02@liv.ac.uk 